\title{
High reflectance of reflective-type attenuated-phase-shifting masks for extreme ultraviolet lithography with high inspection contrast in deep ultraviolet regimes
}

\author{
H. L. Chen ${ }^{\text {a) }}$ \\ Department and Institute of Materials Science and Engineering, National Taiwan University, Taipei, \\ Taiwan, Republic of China \\ H. C. Cheng \\ National Nano Device Laboratory, 1001-1 Ta Hsueh Road Hsinchu, Taiwan, Republic of China \\ T. S. Ko \\ Department of Nuclear Science, National Tsing Hua University, Hsinchu, Taiwan, Republic of China \\ F. H. Ko \\ National Nano Device Laboratory, 1001-1 Ta Hsueh Road, Taiwan, Republic of China \\ T. C. Chu \\ Department of Nuclear Science, National Tsing Hua University, Hsinchu, Taiwan, Republic of China
}

(Received 3 June 2004; accepted 13 September 2004; published 10 December 2004)

\begin{abstract}
Phase-shifting masks are a vital resolution enhance technique that will be used in extreme ultraviolet (EUV) lithography beyond the $20 \mathrm{~nm}$ node. In this article, we demonstrate a structure for a reflective-type attenuated phase-shifting mask, which is based on a Fabry-Perot structure with common materials in EUV masks. The mask structure not only performs $180^{\circ}$ phase shift with high reflectance at EUV wavelength, but also has high inspection contrast at deep ultraviolet (DUV) wavelength. The top layer of mask structures exhibits good conductivity, which can alleviate the charging effect during electron-beam patterning. The reflectance ratio of the absorber stack could be tuned from $32.6 \%\left(\mathrm{TaN} / \mathrm{SiO}_{2} / \mathrm{Mo}\right)$ to $4.4 \%\left(\mathrm{TaN} / \mathrm{SiO}_{2} / \mathrm{TaN}\right)$ by choosing different bottom layers and thickness. The inspection contrast could be raised to $99 \%$ with large thickness-control tolerance. () 2004 American Vacuum Society. [DOI: 10.1116/1.1813450]
\end{abstract}

\section{INTRODUCTION}

Extreme ultraviolet (EUV) lithography is one of the leading candidates for patterning semiconductor devices for sub$50 \mathrm{~nm}$ generations. According to the recent International Technology Roadmap for Semiconductor (ITRS), the resolution enhance techniques (RET) such as phase-shifting-masks (PSM), are also essential in EUV lithography for sub-20 nm nodes. ${ }^{1}$ In deep ultraviolet (DUV) lithography, a high transmittance attenuated phase-shifting mask (HT-APSM) can perform better than a conventional attenuated PSM to improve depth of focus and resolution by destructive optical interference at the edges of features. ${ }^{2}$ Therefore, designing a high reflectance of reflective-type APSM for EUV lithography is imperative.

In recent years, various materials for absorbers and buffer layers have been evaluated for EUV mask applications. ${ }^{3} \mathrm{TaN}$ and $\mathrm{Cr}$ films are the leading choices for absorber materials, and $\mathrm{Si}_{3} \mathrm{~N}_{4}$ and $\mathrm{SiO}_{2}$ films are chosen as the buffer layers. ${ }^{4}$ However, TaN and $\mathrm{Cr}$ films are high reflectance at the inspection wavelength $(257$ or $365 \mathrm{~nm}$ ). The high reflectance decreases the inspection contrast between multilayer mirror and absorber stacks. Therefore, increasing the inspection contrast by decreasing the reflectance of the absorber stack at the inspection wavelength is important. Single-layer dielec-

${ }^{a)}$ Electronic mail: hsuenlichen@ccms.ntu.edu.tw tric antireflective coatings (ARC), such as silicon nitride films, are used to increase the inspection contrast. ${ }^{5}$

Generally, mask layers are patterned by electron-beam lithography. There is an intrinsic electron accumulation problem that causes electron deflection. The problem will become more serious as the feature size of EUV mask decreases to less than $200 \mathrm{~nm} .{ }^{6}$ To avoid the pattern distortion, the mask layer should have good conductivity. The single-layer antireflective coatings, which are dielectric materials with poor conductivity, are not suitable as mask layers for electronbeam patterning.

In this article, we demonstrate a structure for reflectivetype attenuated phase-shifting mask, which is based on a Fabry-Perot structure with common materials of EUV masks. The mask structure not only performs $180^{\circ}$ phase shift with high reflectance at EUV wavelength, but also has high inspection contrast at deep ultraviolet wavelength. The top layer of mask structures exhibits good conductivity, which can alleviate the charging effect during electron-beam patterning. The reflectance ratio between the absorber stack and multilayer mirror could be tuned by choosing different bottom layers and thickness. The inspection contrast could be raised to $99 \%$ with large thickness-control tolerance. The total thickness of the absorber stacks is retained thin enough to meet the stack height requirement to prevent the geometric shadow effect. 
TABLE I. Optical constants of EUVL mask materials at 13.5 and $257 \mathrm{~nm}$ wavelengths.

\begin{tabular}{cccccc}
\hline \hline & \multicolumn{2}{c}{$13.5 \mathrm{~nm}$} & & \multicolumn{2}{c}{$257 \mathrm{~nm}$} \\
\cline { 2 - 3 } \cline { 5 - 6 } & $n$ & $k$ & & $n$ & $k$ \\
\hline $\mathrm{SiO}_{2}$ & 0.97818 & 0.01077 & & 1.50410 & 0.00000 \\
$\mathrm{Si}$ & 0.99932 & 0.00183 & & 1.64026 & 3.91824 \\
$\mathrm{Mo}$ & 0.92125 & 0.00642 & & 1.71573 & 3.74531 \\
$\mathrm{Si}_{3} \mathrm{~N}_{4}$ & 0.97343 & 0.00932 & & 2.24752 & 0.00234 \\
$\mathrm{TaN}$ & 0.92600 & 0.04363 & & 2.49674 & 1.52568 \\
$\mathrm{Cr}$ & 0.93246 & 0.03888 & & 0.86040 & 2.11620 \\
\hline \hline
\end{tabular}

\section{SIMULATION}

In this article, we simulate the optical behavior of a reflective APSM by utilizing the optical multilayer thin film theory. ${ }^{7}$ The optical constants of molybdenum (Mo), silicon ( $\mathrm{Si})$, tantalum nitride $(\mathrm{TaN})$, chromium $(\mathrm{Cr})$, silicon dioxide $\left(\mathrm{SiO}_{2}\right)$, and silicon nitride $\left(\mathrm{Si}_{3} \mathrm{~N}_{4}\right)$ at EUV and DUV wavelengths are obtained from a database by using the method of linear interpolation. ${ }^{8-10}$ Table I shows the optical constants of EUVL mask materials at $13.5 \mathrm{~nm}$ exposure and at $257 \mathrm{~nm}$ inspection wavelengths, respectively.

To characterize the reflectance behaviors, we assume the multilayer (ML) mirror consist of 40-layer pairs of Mo and $\mathrm{Si}$ with layer thickness of 2.8 and $4.1 \mathrm{~nm}$, respectively. The $\mathrm{Mo} / \mathrm{Si}$ ML mirror is deposited on low thermal expansion material substrate and then covered by buffer and absorber layers. We assume the thickness of the buffer to be about $60 \mathrm{~nm}$ to meet the requirement of focused ion beam (FIB) repair stage. The reflectance of ML areas at repair and final stages is controlled at $60 \%$.

Figure 1 shows the phase of two portions of patterned structures, $\varphi_{1}$ and $\varphi_{2}$. The optical path difference (OPD) is defined as the phase shift between the void and the absorber stack. $R_{1}$ and $R_{2}$ are the reflectance of the ML mirror and the absorber stack, respectively. For analysis of the reflectance and phase shift, thin film interference theory was introduced. The exposure conditions used in simulation are $13.5 \mathrm{~nm}$

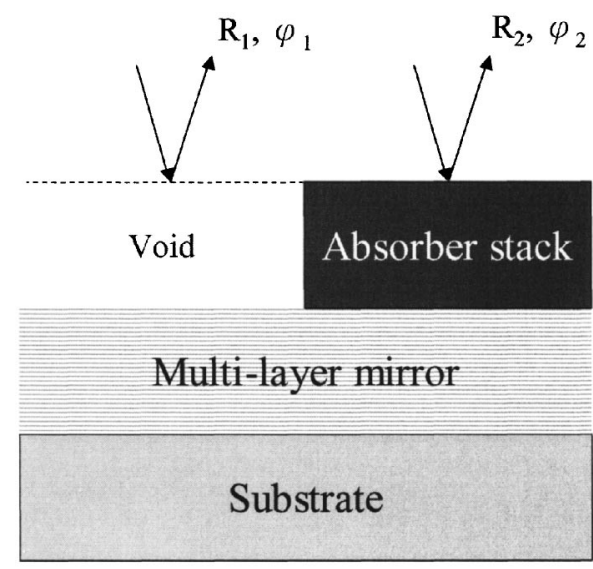

FIG. 1. Diagram of the concept of reflective-type attenuated phase shift mask.

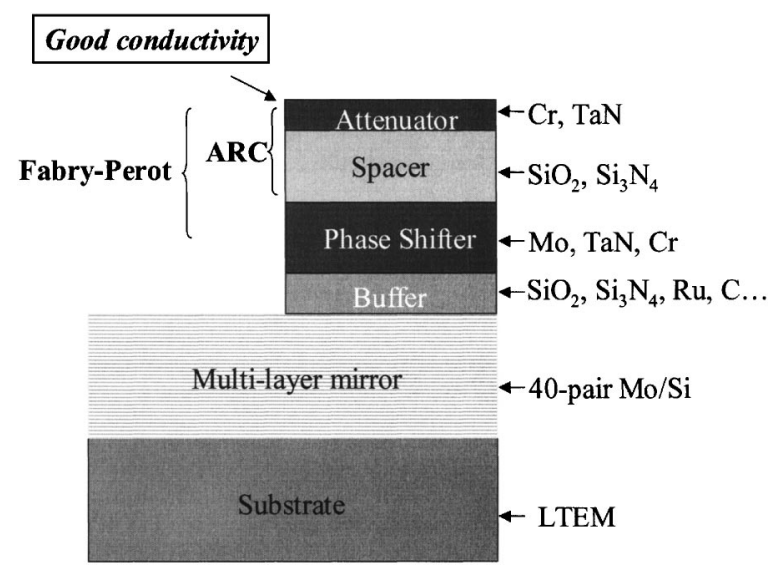

Fig. 2. Diagram of Fabry-Perot-type attenuated phase-shift mask.

wavelength, $s$-polarized, and 5 deg incidence angle.

\section{RESULTS AND DISCUSSION}

\section{A. Absorber stack}

Shown in Fig. 2, is the structure of the absorber stack arranged with buffer, phase shifter, spacer, and attenuator. The buffer can protect the under-ML mirror from damage during etch and repair processes. The phase shifter causes the optical path difference, which has a large refractive index difference to air at the EUV wavelength. The suitable materials for the phase shifter are Mo, TaN, and Cr. The spacer of the Fabry-Perot-type ARC must be a transparent material at the DUV inspection wavelength. The minimum reflectance wavelength can be tuned by tuning the thickness of the spacer. $\mathrm{SiO}_{2}$ and $\mathrm{Si}_{3} \mathrm{~N}_{4}$ are the common materials which meet the spacer requirement. The attenuator is a thin metallic film, such as $\mathrm{TaN}$ or $\mathrm{Cr}$, with suitable optical constants to match the spacer to induce the destructive interference at the DUV inspection wavelength. Based on this concept, we demonstrate the high reflectance APSM composed by the $\mathrm{TaN} / \mathrm{SiO}_{2} / \mathrm{Mo}$ stack, and the normal reflectance APSM composed by the $\mathrm{TaN} / \mathrm{SiO}_{2} / \mathrm{TaN}$ stack.

\section{B. Reflectance and phase shift of absorbers in EUV wavelength region}

Figure 3 shows the reflectance and phase shift of the $\mathrm{TaN} / \mathrm{SiO}_{2} / \mathrm{Mo}$ stack at $13.5 \mathrm{~nm}$. The thickness of the TaN layer and $\mathrm{SiO}_{2}$ layer is fixed at 4.4 and $27.2 \mathrm{~nm}$, respectively, to maintain the good DUV inspection contrast. As the Mo layer thickness increases, the reflectance of the absorber stack is varied sinusoidally with a small decrease, and the relative phase shift is increased linearly. As the $180^{\circ}$ phase shift thickness of the Mo layer is $30.1 \mathrm{~nm}$, and the reflectance ratio $\left(R_{1} / R_{1}\right)$ is $32.6 \%$ at $13.5 \mathrm{~nm}$, the structure can satisfy the requirements of high reflectance APSM. Figure 3 also shows that phase shift is $180^{\circ} \pm 10^{\circ}$ as the thickness of Mo layer ranges from 29 to $35 \mathrm{~nm}^{5}$

Similarly, Fig. 4 illustrates the reflectance and phase shift of a low reflectance APSM structure composed with the 


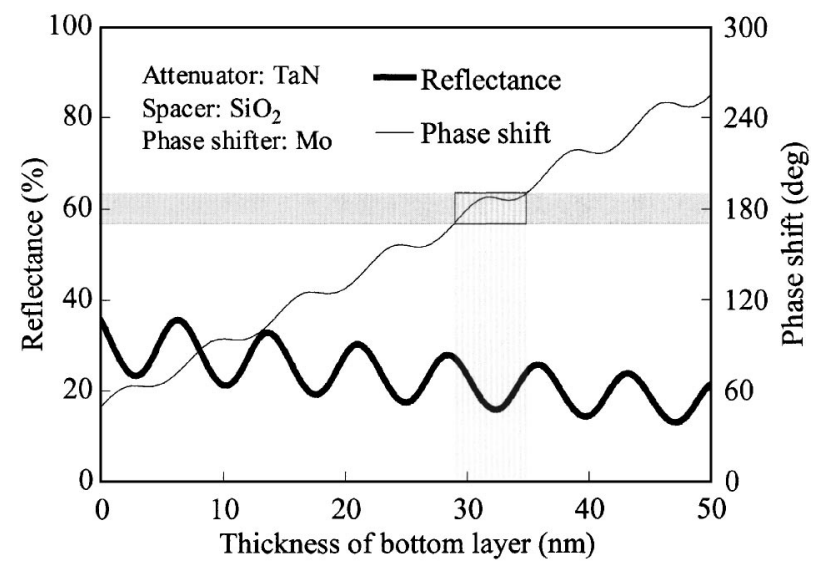

FIG. 3. Reflectance and phase shift of $\mathrm{TaN} / \mathrm{SiO}_{2} / \mathrm{Mo}$ stack structure at $13.5 \mathrm{~nm}$.

$\mathrm{TaN} / \mathrm{SiO}_{2} / \mathrm{TaN}$ absorber stack. The thickness of the top TaN layer and $\mathrm{SiO}_{2}$ layer is fixed at 1.9 and $31.8 \mathrm{~nm}$, respectively, to get good DUV inspection contrast. As the thickness of the bottom TaN film is increased, the reflectance of the absorber stack decreases rapidly with small ripples, and the phase shift is increased linearly. This is different from the previous high reflectance APSM structure because the extinction coefficient of a TaN film is one order larger than a Mo film in extreme ultraviolet regimes. At $180^{\circ}$ phase shift, the thickness of the bottom TaN films is $30.4,35.7$, and $40.7 \mathrm{~nm}$, and the corresponding reflectance ratio $\left(R_{2} / R_{1}\right)$ is $4.4 \%, 4.3 \%$, and $0.9 \%$ at $13.5 \mathrm{~nm}$.

\section{Reflectance of absorbers in DUV inspection wavelength region}

The reflectance of ML mirror is about $60 \%$ in the DUV regime. The reflectance of EUV mask absorber stacks such as $\mathrm{TaN}$ or $\mathrm{Cr}$ is larger than $30 \%$. Therefore, decreasing the reflectance of the absorber stack to increase the inspection contrast is essential. In this article, by controlling the thickness of the spacer and top attenuator layers, the reflectance of the absorber stack can be decreased to less than $1 \%$. Figure 5

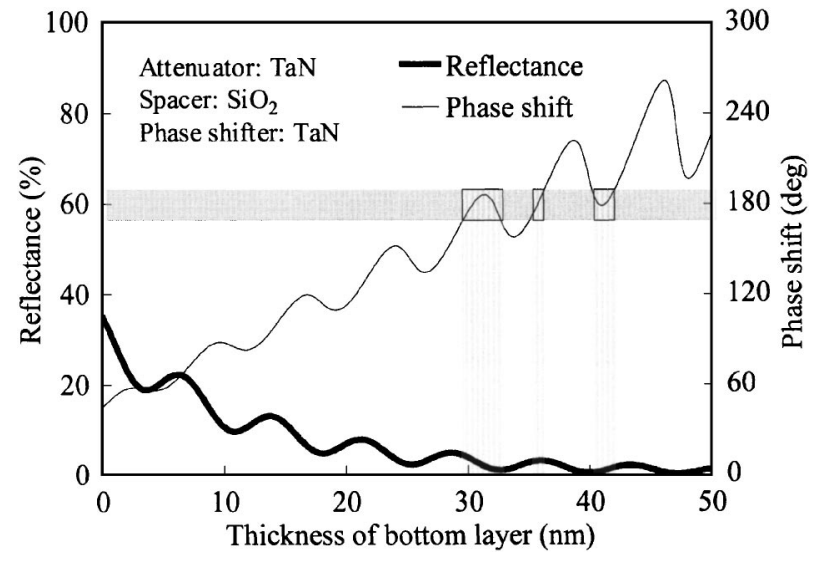

FIG. 4. Reflectance and phase shift of $\mathrm{TaN} / \mathrm{SiO}_{2} / \mathrm{TaN}$ stack structure at $13.5 \mathrm{~nm}$.

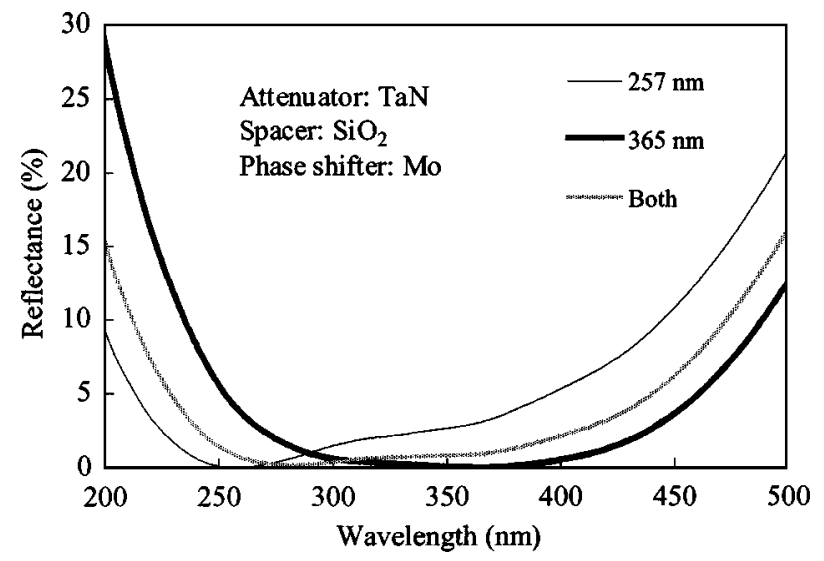

FIG. 5. Reflectance of $\mathrm{TaN} / \mathrm{SiO}_{2} / \mathrm{Mo}$ absorber stack in the inspection wavelength regime.

demonstrates the reflectance of $\mathrm{TaN} / \mathrm{SiO}_{2} / \mathrm{Mo}$ absorber stack in the DUV inspection regime. Like the $4.4 \mathrm{~nm}$ of the top TaN layer and $27.2 \mathrm{~nm}$ of the $\mathrm{SiO}_{2}$ layer, the reflectance can be reduced to less than $1 \%$ at $257 \mathrm{~nm}$. We can also tune the thickness of $\mathrm{SiO}_{2}$ and top TaN films to shift the minimal reflectance regime to $365 \mathrm{~nm}$ or another desired inspection wavelength.

Similarly, Fig. 6 shows the reflectance of $\mathrm{TaN} / \mathrm{SiO}_{2} / \mathrm{TaN}$ absorber stack in the inspection wavelength regime. The reflectance is lower than $1 \%$ at $257 \mathrm{~nm}$, like the thickness $1.9 \mathrm{~nm}$ of top TaN layer and $31.8 \mathrm{~nm}$ of $\mathrm{SiO}_{2}$ layer. We can also tune the thickness of the absorber structure for another inspection wavelength.

\section{Inspection contrast and thickness variation tolerance}

The inspection contrast is less than $30 \%$ between the ML mirror and conventional $\mathrm{TaN}$ and $\mathrm{Cr}$ absorber stacks without adding antireflective coatings. The inspection contrast should be larger than $40 \%$ for defect identification. ${ }^{11}$ Figure 7 shows the inspection contrast depending on the thickness variation of the $\mathrm{TaN} / \mathrm{SiO}_{2} / \mathrm{Mo}$ absorber stack. With as the change of thickness, the inspection contrast decreases. The $\mathrm{SiO}_{2}$ layer

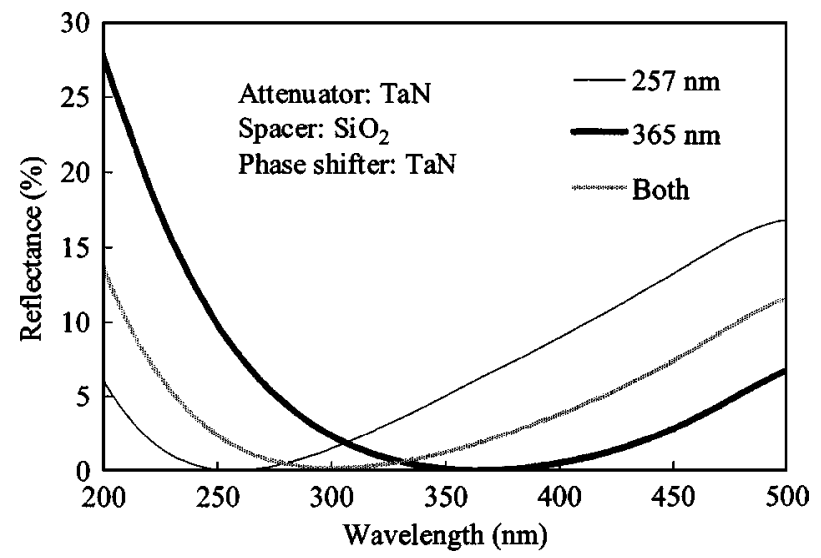

FIG. 6. Reflectance of $\mathrm{TaN} / \mathrm{SiO}_{2} / \mathrm{TaN}$ absorber stack in the inspection wavelength regime. 


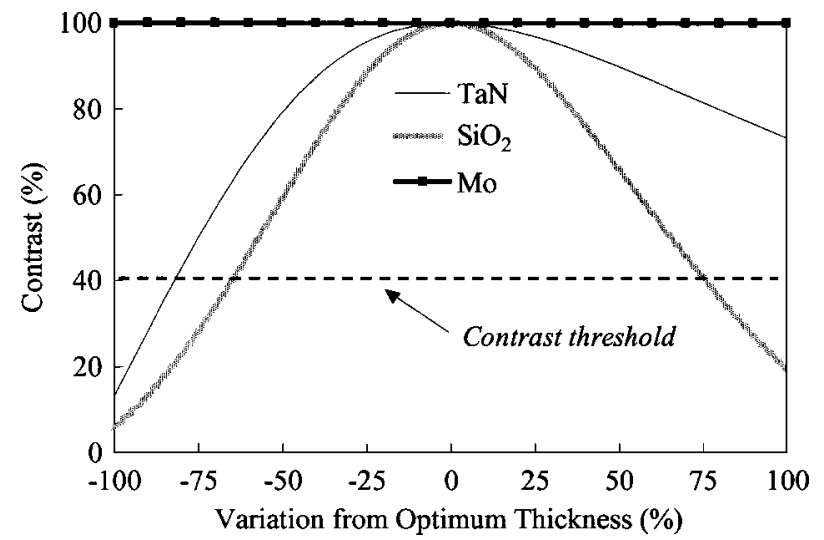

FIG. 7. Inspection contrast depending on the variation of film thickness in the $\mathrm{TaN} / \mathrm{SiO}_{2} / \mathrm{Mo}$ absorber stack.

is found to be the most sensitive layer in the absorber structure. The inspection contrast can remain larger than $40 \%$ as the thickness variation of the $\mathrm{SiO}_{2}$ layer ranges from $-63 \%$ to $75 \%$.

Similarly, the inspection contrast depending on the variation of $\mathrm{TaN} / \mathrm{SiO}_{2} / \mathrm{TaN}$ thicknesses is shown in Fig. 8. The most sensitive $\mathrm{SiO}_{2}$ layer is shown with $-85 \%-88 \%$ thickness-variation-tolerance range. For the large thickness-

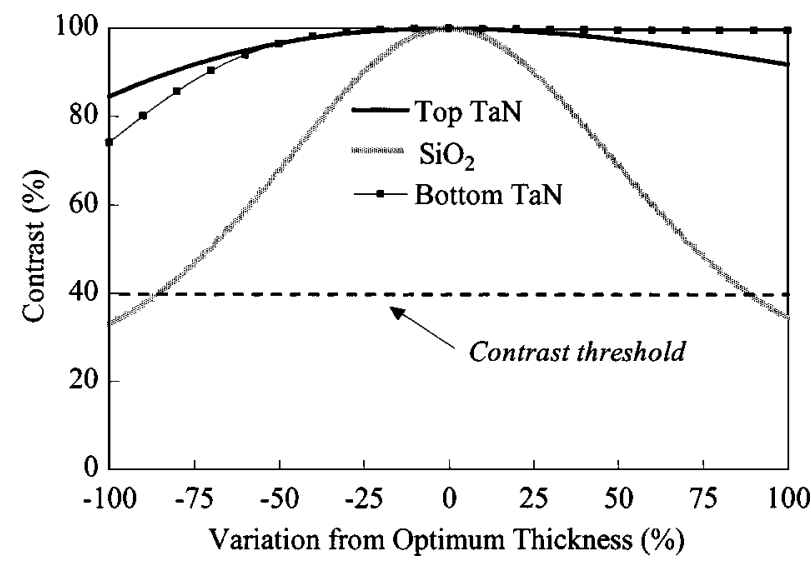

FIG. 8. Inspection contrast depending on the variation of film thickness in the $\mathrm{TaN} / \mathrm{SiO}_{2} / \mathrm{TaN}$ absorber stack. tolerance range, we can tune the thickness of the absorber structure to meet the requirements of reflectance ratio and phase shift in the EUV regime for reflective-type APSM applications.

\section{CONCLUSIONS}

In this article, we demonstrated a structure for a reflective-type attenuated phase-shifting mask, which is based on a Fabry-Perot structure with common materials of EUV masks. The mask structure not only performs $180^{\circ}$ phase shift with high reflectance at EUV wavelength, but also has high inspection contrast at deep ultraviolet wavelength. The top layer of mask structures exhibits good conductivity, which can alleviate the charging effect during electron-beam patterning. The reflectance ratio of the absorber stack could be tuned from $32.6 \%\left(\mathrm{TaN} / \mathrm{SiO}_{2} / \mathrm{Mo}\right)$ to $4.4 \%\left(\mathrm{TaN} / \mathrm{SiO}_{2} / \mathrm{TaN}\right)$ by choosing different bottom layers and thickness. The inspection contrast could be raised to $99 \%$ with large thicknesscontrol tolerance. For the large thickness-tolerance range, we can tune the thickness of the absorber structure to meet the requirements of reflectance ratio and phase shift in the EUV regime for various reflective-type APSM applications. The total thickness of the absorber stacks is less than $80 \mathrm{~nm}$ to meet the stack height requirement to prevent the geometric shadow effect.

\footnotetext{
${ }^{1}$ Semiconductor Industry Association, International Technology Roadmap for Semiconductor 2003 Updated (SIA Publication, 2003).

${ }^{2}$ F. D. Lai, C. M. Chang, L. A. Wang, and T. S. Yih, J. Vac. Sci. Technol. B 21, 3062 (2003).

${ }^{3}$ S. D. Hector, Proc. SPIE 4688, 134 (2002).

${ }^{4}$ K. H. Smith, J. R. Wasson, P. J. S. Mangat, W. J. Dauksher, and D. J. Resnick, J. Vac. Sci. Technol. B 19, 2906 (2001).

${ }^{5}$ S. I. Han, J. R. Wasson, P. J. S. Mangat, J. L. Cobb, K. Lucas, and S. D. Hector, Proc. SPIE 4688, 481 (2002).

${ }^{6}$ B. Lu, J. R. Wasson, E. J. Weisbrod, Z. Masnyj, P. J. S. Mangat, K. Nordquist, and D. Resnick, J. Vac. Sci. Technol. B 20, 3029 (2002).

${ }^{7}$ H. A. Macleod, Thin-Film Optical Filters, 2nd ed. (Hilger, London, 1986).

${ }^{8}$ B. L. Henke, E. M. Gullikson, and J. C. Davis, At. Data Nucl. Data Tables 54, 181 (1993)

${ }^{9}\langle$ http://www.cxro.lbl.gov/optical_constants/〉.

${ }^{10}$ Edward D. Palik, Handbook of Optical Constants of Solids (Academic, New York, 1998).

${ }^{11}$ S. Hector and P. Mangat, J. Vac. Sci. Technol. B 19, 2612 (2001).
} 